\title{
Erratum: Lbx 1 and T/x3 are opposing switches in determining GABAergic versus glutamatergic transmitter phenotypes
}

Leping Cheng, Omar Abdel Samad, Yi Xu, Rumiko Mizuguchi, Ping Luo, Senji Shirasawa, Martyn Goulding \& Qiufu Ma Nat. Neurosci. 8, 1510-1515 (2005)

This article contained a misspelling. Lhx1/2 should have read Lhx1/5 throughout the text.

\section{Erratum: Why pictures look right when viewed from the wrong place}

Dhanraj Vishwanath, Ahna R Girshick \& Martin S Banks

Nat. Neurosci. 8, 1401-1410 (2005)

On page 1402, the first two sentences of the second full paragraph in the second column were omitted. The paragraph should have begun as follows: "An alternative explanation, the local-slant hypothesis, suggests that location of the CoP is not recovered. Instead, the observed invariance is due to an adjustment of the retinal-image shape based on measurements of the local slant of the picture surface at the point of interest. This hypothesis does not require estimates of the location of or distance to the CoP."

\section{Erratum: Top-down suppression deficit underlies working memory impair- ment in normal aging}

\section{Adam Gazzaley, Jeffrey W Cooney, Jesse Rissman \& Mark D’Esposito}

Nat. Neurosci. 8, 1298-1300 (2005)

The article contained typographical errors in the labeling of the $y$ axes in Figures $2 \mathrm{~b}$ and $2 \mathrm{c}$. The label for the $y$ axis on Figure $2 \mathrm{~b}$ should read $\beta$, and the label for the $y$ axis on Figure $2 \mathrm{c}$ should read $\Delta \beta$.

CORRIGENDUM

\section{Corrigendum: Orexin, drugs and motivated behaviors}

\section{Thomas E Scammell \& Clifford B Saper}

Nat. Neurosci. 8, 1286-1288 (2005)

This article contains an incorrect citation. The name of the last author in reference 2 ("A role for lateral hypothalamic orexin neurons in reward seeking" Nature 2005) should read Aston-Jones, G., rather than Jones, G.A. The authors regret the error. 